\title{
Effect of postural change on shoulder joint internal and external rotation range of motion in healthy adults in their $20 \mathrm{~s}$
}

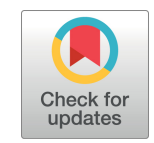

\author{
Beom-Ryong Kim ${ }^{\mathrm{a}, \mathrm{b}}$, Dong-Hyun $\mathrm{Yi}^{\mathrm{b}}$, Jong-Eun Yim ${ }^{\mathrm{b}, \mathrm{c}}$ \\ ${ }^{a}$ Department of Physical Therapy, Design Hospital, Jeonju, Republic of Korea \\ ${ }^{b}$ Department of Physical Therapy, The Graduate School of Sahmyook University, Seoul, Republic of Korea \\ ${ }^{\mathrm{c} D e p a r t m e n t ~ o f ~ P h y s i c a l ~ T h e r a p y, ~ C o l l e g e ~ o f ~ H e a l t h ~ S c i e n c e ~ a n d ~ S o c i a l ~ W e l f a r e, ~ S a h m y o o k ~ U n i v e r s i t y, ~ S e o u l, ~ R e p u b l i c ~ o f ~ K o r e a ~}$
}

Objective: We aimed to investigate differences of range of motion in measuring shoulder internal rotation (IR) and external rotation (ER) resulting from posture change in manual scapular stabilization in prone, hook-lying, sitting, and standing positions in healthy young adults.

Design: Cross-sectional study.

Methods: This study included healthy young adults who agreed to participate after a thorough explanation about the study purpose and methods. A clinometer was used to measure shoulder rotation. Measurements of shoulder rotation according to postural change were performed in prone, hook-lying, sitting, and standing positions. The repeated measures analysis of variance was used to compare between-group differences in postural change.

Results: The lower the posture, the greater the average value of IR angle. In contrast, the higher the posture, the greater the average value of the external rotation angle. In active and passive IR with posture change, there was difference in average value but with no statistical significance. In active and passive ER, there was a statistically significant difference between prone and sitting, prone and standing, hook-lying and sitting, hook-lying and standing, and sitting and standing position $(p<0.05)$.

Conclusions: Our findings suggest that postural change should be considered in order to increase the strength or range of motion of the internal and external rotation of the patient's shoulder joint.

Key Words: External rotation, Internal rotation, Shoulder, Range of motion

\section{Introduction}

Range of motion (ROM) is the amount of movement of a joint measured when moving the legs and arms. This is an important indicator in the assessment of a medical disorder or to assess the degree of improvement of an intervention $[1,2]$. Therefore, the measurement of limited ROM can be regarded as one of the important clinical test methods for assessing disorder of intra-articular or surrounding tissue lesions or the effectiveness of interventions [3].

The scapulothoracic joint of the shoulder complex performs the appropriate scapula movement by cooperative movements with the sternoclavicular joint and the acromio- clavicular joint, and this affects the movement of the humerus in the glenohumeral joint. Therefore, the joints of the shoulder complex are intimately interconnected to facilitate maximum shoulder movement. In addition, there are 16 muscles attached to the shoulder complex, and the range of shoulder movement is controlled by these muscles. However, if one of these joints experiences a problem or one of the associated muscles is weakened, the movement control of the shoulder complex may be reduced to produce an abnormal shoulder movement, which limits the movement of the shoulder [4]. Shoulder disease typically include rotator cuff tear or shoulder impingement syndrome. Such shoulder disease causes limited internal rotation (IR) and external ro-

Received: 2 September, 2019 Revised: 17 September, 2019 Accepted: 17 September, 2019

Corresponding author: Jong-Eun Yim (ORCID https://orcid. org/0000-0001-7510-8233)

Department of Physical Therapy, College of Health Science and Social Welfare, Sahmyook University, 815 Hwarang-ro, Nowon-gu, Seoul 01795, Republic of Korea Tel: 82-2-3399-1635 Fax: 82-2-3399-1639 E-mail: jeyim@syu.ac.kr

(c) This is an Open-Access article distributed under the terms of the Creative Commons Attribution Non-Commercial License (http://creativecommons.org/licenses/ by-nc/4.0) which permits unrestricted non-commercial use, distribution, and reproduction in any medium, provided the original work is properly cited.

Copyright (๑) 2019 Korean Academy of Physical Therapy Rehabilitation Science 
tation (ER) ROM of the shoulder joint [5]. Therefore, a clinical assessment of the rotation of the shoulder is needed.

To measure shoulder rotation ROM, a goniometer was used to assess elite junior tennis players [6,7] and baseball players [8-10] in the hook-lying position, the shoulder in $90^{\circ}$ of abduction, the elbow at $90^{\circ}$ of flexion. Assuming a hooklying position is the general assessment procedure in measuring ROM shoulder rotation. According to Sadeghifar et al. [11], another method of measuring shoulder rotation ROM involves using a goniometer in standing position with the shoulder in $90^{\circ}$ abduction and elbow in $90^{\circ}$ flexion for persons with shoulder dislocation. Sadeghifar et al. [11] also measures shoulder rotation in standing position so that IR is aided by gravity. ER rises against gravity up to a range of $90^{\circ}$, but it is aided by gravity beyond $90^{\circ}$. Previous studies have applied manual scapular stabilization that minimizes the movement of scapulothoracic joints in hook-lying or standing position to accurately measure ROM of shoulder rotation [12]. However, the measurements in these previous studies did not take into account the potential effect of gravity upon posture change for shoulder movement. Joint movement with gravity is thought to increase the measurement value of ROM by applying passive forces. Keating and Matyas [13] showed differences in the muscular strength of the knee extensor resulting from posture changes during knee extension operation.

In this study, we aimed to investigate differences of shoulder IR and ER ROM resulting from postural change in manual scapular stabilization in prone, hook-lying, sitting, and standing positions in healthy young adults.

\section{Methods}

\section{Participants}

This study included practice students who visited Design Hospital rehabilitation center in Jeonju in April 2019. The purpose and method of the experiment were described thoroughly, and those who agreed to participate in the experiment were selected. The Anterior Slide test was performed to find shoulder pain [14]. The Anterior and Posterior Drawer Tests were performed to determine anterior and posterior shoulder instability [15]. A positive Sulcus sign would indicate inferior shoulder instability [16]. Those with chronic shoulder pain or persistently severe pain, neuropathy, or anyone who was found to have had sensory abnormalities or muscle paralysis, those with difficulty in exercise performance ability due to possible mental challenges or lack of cognitive understanding were excluded from the study. The general characteristics of the subjects are shown in Table 1.

This study was approved by the Institutional Review Board of the Sahmyook University (IRB no. 2-7001793AB-N-012019015HR) and informed consent was obtained.

\section{Outcome measurements and procedure}

An Android smartphone (Samsung Galaxy S7, Samsung Electronics Co., Ltd., Suwon, Korea) was used to measure shoulder rotation using an application that was downloaded from Google Play named Smartphone Clinometer Application (Plaincode Software Solutions, Stephanskirchen, Germany). The clinometer is an easy to use, low-cost solution available on many smartphones. The evaluator downloaded the clinometer to the smartphone, configured it, and became familiar with its functionality before measurement. Measurements of shoulder rotation according to postural change were per-

Table 1. General characteristics of the participants

$(\mathrm{N}=9)$

\begin{tabular}{cccccc}
\hline Subjects & Sex & Age $(\mathrm{y})$ & Height $(\mathrm{cm})$ & Weight $(\mathrm{kg})$ & Body mass index $\left(\mathrm{kg} / \mathrm{cm}^{2}\right)$ \\
\hline 1 & Female & 23 & 163 & 47 & 17.69 \\
2 & Female & 22 & 171 & 58 & 19.84 \\
3 & Female & 22 & 155 & 46 & 19.15 \\
4 & Female & 22 & 171 & 64 & 21.89 \\
5 & Female & 22 & 161 & 56 & 21.6 \\
6 & Female & 22 & 165 & 47 & 17.26 \\
7 & Male & 23 & 164 & 50 & 18.59 \\
8 & Female & 22 & 165 & 58 & 21.3 \\
9 & Female & 22 & 158 & 57 & 22.83 \\
Mean (SD) & & $22.22(0.44)$ & $163.67(5.31)$ & $53.67(6.34)$ & $20.02(1.98)$ \\
\hline
\end{tabular}

Values are presented as number only or mean (SD). 


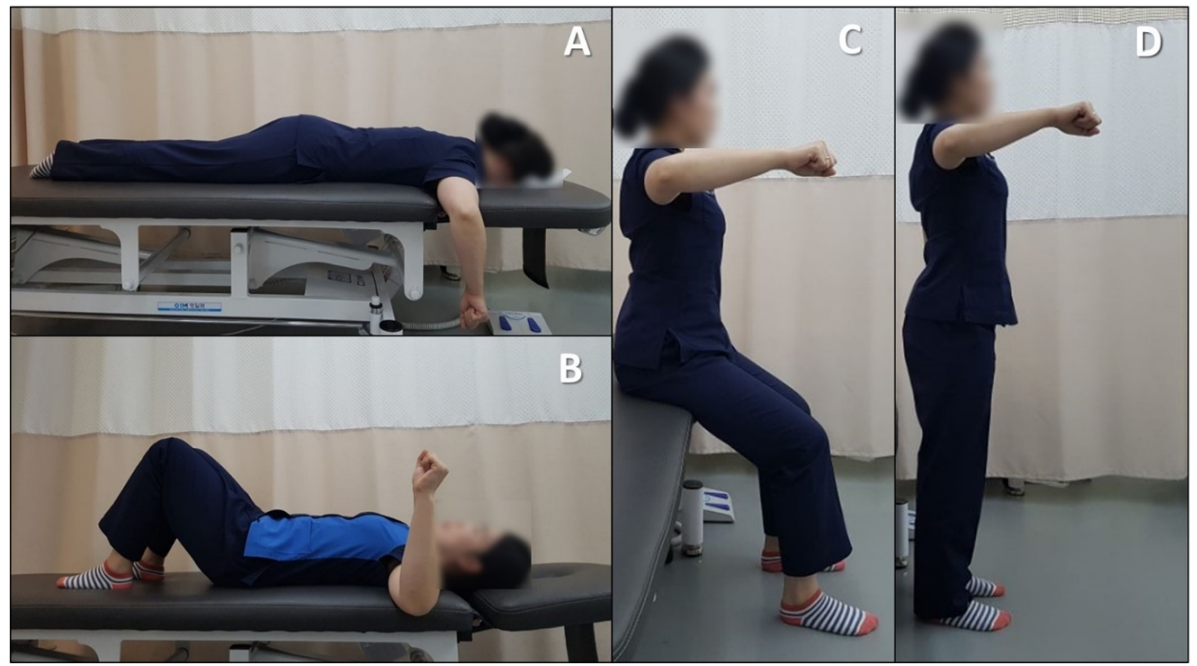

Figure 1. Measurement position. (A) Prone. (B) Hook lying. (C) Sitting. (D) Standing.

Table 2. Comparison of internal and external rotation angle by position

$(\mathrm{N}=9)$

\begin{tabular}{lcccc}
\hline \multicolumn{1}{c}{ Position } & AIR & PIR & AER & PER \\
\hline Prone $\left(^{\circ}\right)$ & $56.50(8.38)$ & $65.72(6.04)$ & $89.83(7.69)$ & $98.28(7.25)$ \\
Hook-lying $\left(^{\circ}\right)$ & $55.55(7.56)$ & $64.72(8.64)$ & $90.17(12.63)$ & $100.17(13.43)$ \\
Sitting $\left({ }^{\circ}\right)$ & $55.17(9.32)$ & $64.17(9.34)$ & $100.11(10.56)$ & $110.72(11.93)$ \\
Standing $\left({ }^{\circ}\right)$ & $54.78(7.49)$ & $63.50(9.71)$ & $103.44(10.54)$ & $112.55(11.00)$ \\
$\mathrm{F}(p)$ & $0.246(0.864)$ & $0.729(0.405)$ & $29.343(<0.001)$ & $28.759(<0.001)$ \\
\hline
\end{tabular}

Values are presented as mean (SD).

AIR: active internal rotation, PIR: passive internal rotation, AER: active external rotation, PER: passive external rotation.

formed in prone, hook-lying, sitting, and standing positions (shoulder $90^{\circ}$ abduction, elbow $90^{\circ}$ flexion) with manual scapular stabilization on the treatment table [12]. Measurement positions are shown in Figure 1. The patient was asked to have the back and buttocks touch the wall to prevent trunk motion for the sitting and standing shoulder rotation measurements. The clinometer measured the angle in parallel with the ulna. The clinometer measurements were obtained by reading the value on the screen. The average value was recorded by repeating the measurement three times. Inter-rater reliability is an appropriate tool for measuring ROM intra-class correlation coefficient (ICC) for the clinometer [17].

\section{Statistical analysis}

Statistical analysis was performed using PASW Statistics for Windows, Version 18.0 (SPSS Inc., Chicago, IL, USA). The repeated measures ANOVA was used to compare the between-group differences for postural change. Statistical significance was set at $p<0.05$.

\section{Results}

The IR and ER angles of the shoulder joint with postural change are shown in Table 2 and Figure 2. The lower the posture, the greater the average value of IR angles. In contrast, the higher the posture, the greater the average value of the ER angles.

In active IR measurement, the highest angle was in the order of prone, hook-lying, sitting, and standing positions. In active IR with posture change, there was difference in average value but with no statistical significance. In passive IR measurement, the highest angle was in the order of prone, hook-lying, sitting, and standing positions. In passive IR with posture change, there was difference in average value but with no statistical significance.

In active ER measurement, the highest angle was shown in the order of standing, sitting, hook-lying, and prone positions. There was a statistically significant difference between prone and sitting, prone and standing, hook-lying and sitting, hook-lying and standing, and sitting and standing $(p<0.05)$ positions. In passive ER measurement, the highest 
A
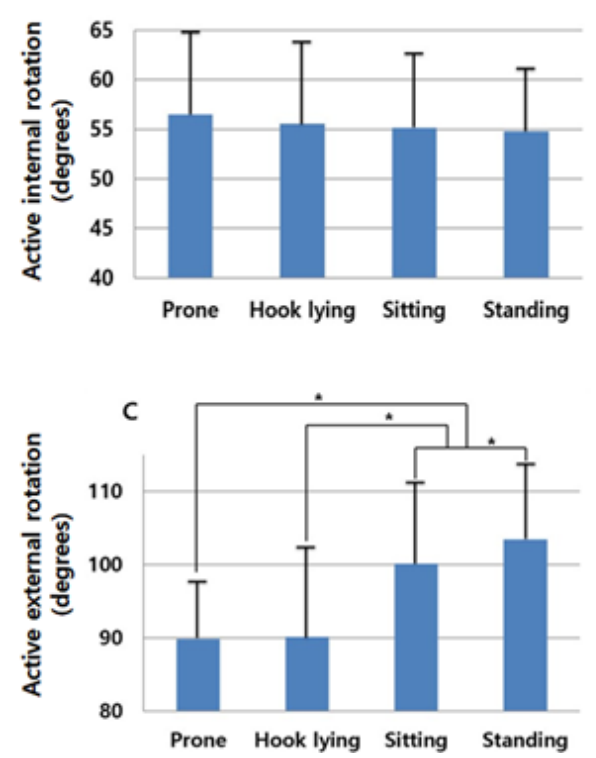

B
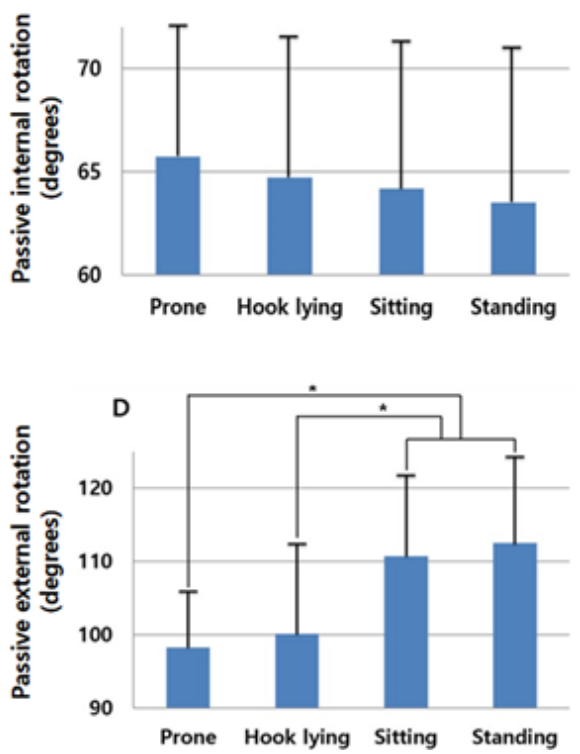

Figure 2. Comparison of internal and external rotation external rotation angle (in ${ }^{\circ}$ ) by position. (A) Active internal rotation. (B) Passive internal rotation. (C) Active external rotation. (D) Passive external rotation. *Significant difference between groups (repeated measures analysis of variance). angle was shown in the order of standing, sitting, hook-lying, and prone positions. There was a statistically significant difference between prone and sitting, prone and standing, hook-lying and sitting, hook-lying and standing $(p<0.05)$ positions.

\section{Discussion}

One of the considerations for measuring shoulder ROM using a goniometer or a clinometer is the posture of the subject during the measurement. The functional activity of the upper extremity is mostly performed in the sitting position [18], however in the actual clinic with stroke survivors, the ROM of the shoulder is measured in the hook-lying position in most cases. It is easy to control the trunk or scapular adduction and provides a wide base of support (BOS) to allow accurate ROM measurements by decreasing the tone of muscles with increased tone. In this study, we investigated the differences of shoulder rotation according to postural change. Rotation was measured in various postures, from the prone position (high trunk weight support), which provides a wide BOS, to standing position (low trunk weight support), which provides a narrow BOS. As a result, the average value of IR angle increased with lower postures. In contrast, the higher the posture, the greater the average values of the ER angle.

The biggest advantage when using a clinometer is the convenience. When measuring ROM with a goniometer, stabilization is performed by having the therapist use one hand of on the fixed arm and the other hand used to move the moving arm [19]. Because the measurement posture is unstable, errors can occur as the axis and fixed arm move, and it is difficult to maintain the moving arm exactly parallel to the length of the arm as it follows the movement. Also, the reading accuracy of the goniometer scale is slow and time consuming. However, when using the clinometer, the error of measurement from an unstable measurement posture is reduced because the smartphone is attached to the patient with the armband, and the clinometer can minimize error because it does not need the imaginary vertical line required for measurement. Also, the measured angle is expressed as a number on the smartphone monitor in real-time, and an accurate measurement is immediately possible [20]. Yang et al. [21] measured hip joint ROM using a clinometer for 50 normal adults. Studies have shown that the inter-rater reliability ( $\mathrm{ICC}=0.95-0.97)$ of the clinometer is higher than the inter-rater reliability $(\mathrm{ICC}=0.78-0.83$ ) of the goniometer. Werner et al. [17] measured the ROM of shoulder rotation using a goniometer and a clinometer for normal adults. Studies have shown that the inter-rater reliability of the goniometer has a low inter-rater reliability with IR $(\mathrm{ICC}=0.64)$ and $\mathrm{ER}$ ( $\mathrm{ICC}=0.78-0.83$ ). However, the inter-rater reliability through the clinometer with $\mathrm{IR}(\mathrm{ICC}=0.81)$ and $\mathrm{ER}$ $(\mathrm{ICC}=0.86)$ was higher inter-compared with the goniometer. In this study, the measurement of shoulder rotation in normal adults was performed using a clinometer with inter-rater reliability confirmed in previous studies.

ROM measurement is one of several tests performed dur- 
ing a clinical visit, and is particularly suitable for testing mobility [22]. Because the shoulder is the most widely moving joint on the body and therefore can have the greatest functional impact, it is very important to have an accurate measurement of the ROM [23,24]. In this study, IR measurements showed the highest angle in prone, which is the lowest posture, followed by hook-lying, sitting, and standing positions. ER measurements showed the highest angle at the highest posture, which was standing, followed by sitting, hook-lying, and prone positions. These results showed that the angle of IR was high when assuming a low posture with a wide BOS. Correspondingly, ER was high when assuming a high posture with a narrow BOS. Stability is needed to increase mobility. In IR motion, scapular protraction occurs and scapular stability decreases. In ER motion, scapular retraction occurs and scapular stability increases [25]. The scapula should be stabilized when measuring shoulder rotation. However, due to previous studies, IR has the largest angle at low postures, where scapular stability is the highest. ER appears to have the largest angle at high postures, where scapular stability is the greatest. In previous studies, specific exercises were designed for the push-up, in which the shoulder is in IR or neutral position, with the palm is facing downwards [26-29]. In this exercise, the push-up modification emphasizing IR seems to be performed in a low posture with wide BOS. A wall-slide exercise, which emphasizes ER, may be performed in a high posture with narrow BOS. In addition, when performing exercise for ROM increase of IR, BOS should be performed at wide, low postures. When performing exercise for ROM increase of ER, BOS should be performed at narrow, high postures. Also, at the anatomical location, the IR and ER of the shoulder joints are related to the roll and slide arthrokinematics. However, shoulder rotation at approximately $90^{\circ}$ of abduction position of this study is good for visualizing kinematics because spin between one point of the head of the humerus and one point of the articular fossa occurs. Therefore, measurement of shoulder rotation in approximately $90^{\circ}$ of abduction according to postural change of this study may be useful for patient intervention and assessment.

There are some limitations to this study. First, measurement of shoulder rotation in side-lying position should be performed with the goniometer because there is difficulty in measuring shoulder rotation in the side-lying position using a clinometer. Second, the measurement of shoulder rotation was measured in the $90^{\circ}$ abduction position. Therefore, an- other method of measurement of shoulder rotation is needed. Finally, although statistically significant results were obtained, the number of subjects is not sufficient to generalize the results of the study, and caution is needed in generalizing the study results to the majority of healthy young adult women.

In conclusion, the purpose of this study was to evaluate the ROM of the shoulder according to postural change in healthy young adults. The results of the study showed that the lower the posture, the higher the angle of IR, and the higher the posture the higher the angle of the ER. Therefore, it is considered that postural change should be considered in order to increase the strength of the shoulder or the IR and ER of the shoulder.

\section{Conflict of Interest}

The authors declared no potential conflicts of interest with respect to the authorship and/or publication of this article.

\section{References}

1. Laudner KG, Sipes RC, Wilson JT. The acute effects of sleeper stretches on shoulder range of motion. J Athl Train 2008;43: 359-63.

2. Shanley E, Rauh MJ, Michener LA, Ellenbecker TS, Garrison JC, Thigpen CA. Shoulder range of motion measures as risk factors for shoulder and elbow injuries in high school softball and baseball players. Am J Sports Med 2011;39:1997-2006.

3. Borsa PA, Dover GC, Wilk KE, Reinold MM. Glenohumeral range of motion and stiffness in professional baseball pitchers. Med Sci Sports Exerc 2006;38:21-6.

4. Neumann DA. Kinesiology of the musculoskeletal system e-book: foundations for rehabilitation. Elsevier Health Sciences; 2013.

5. Lewis JS, Wright C, Green A. Subacromial impingement syndrome: the effect of changing posture on shoulder range of movement. J Orthop Sports Phys Ther 2005;35:72-87.

6. Ellenbecker TS, Roetert EP, Piorkowski PA, Schulz DA. Glenohumeral joint internal and external rotation range of motion in elite junior tennis players. J Orthop Sports Phys Ther 1996;24:336-41.

7. McConnell J, McIntosh B. The effect of tape on glenohumeral rotation range of motion in elite junior tennis players. Clin $\mathrm{J}$ Sport Med 2009;19:90-4.

8. Scher S, Anderson K, Weber N, Bajorek J, Rand K, Bey MJ. Associations among hip and shoulder range of motion and shoulder injury in professional baseball players. J Athl Train 2010;45:191-7.

9. Sauers EL, Huxel Bliven KC, Johnson MP, Falsone S, Walters S. Hip and glenohumeral rotational range of motion in healthy professional baseball pitchers and position players. Am J Sports 
Med 2014;42:430-6.

10. Oliver GD, Weimar WH. Hip and shoulder range of motion in youth baseball pitchers. J Strength Cond Res 2016;30:2823-7.

11. Sadeghifar A, Ilka S, Dashtbani H, Sahebozamani M. A comparison of glenohumeral internal and external range of motion and rotation strength in healthy and individuals with recurrent anterior instability. Arch Bone Jt Surg 2014;2:215-9.

12. Boon AJ, Smith J. Manual scapular stabilization: its effect on shoulder rotational range of motion. Arch Phys Med Rehabil 2000;81:978-83.

13. Keating JL, Matyas TA. Method-related variations in estimates of gravity correction values using electromechanical dynamometry: a knee extension study. J Orthop Sports Phys Ther 1996;24: 142-53.

14. Kibler WB. Specificity and sensitivity of the anterior slide test in throwing athletes with superior glenoid labral tears. Arthroscopy 1995;11:296-300.

15. Gerber C, Ganz R. Clinical assessment of instability of the shoulder. With special reference to anterior and posterior drawer tests. J Bone Joint Surg Br 1984;66:551-6.

16. McFarland EG, Campbell G, McDowell J. Posterior shoulder laxity in asymptomatic athletes. Am J Sports Med 1996;24:46871.

17. Werner BC, Holzgrefe RE, Griffin JW, Lyons ML, Cosgrove CT, Hart JM, et al. Validation of an innovative method of shoulder range-of-motion measurement using a smartphone clinometer application. J Shoulder Elbow Surg 2014;23:e275-82.

18. Radomski MV, Latham CAT. Occupational therapy for physical dysfunction. Philadelphia: Lippincott Williams and Wilkins; 2008.

19. Clarkson HM. Musculoskeletal assessment: joint range of motion and manual muscle strength. Philadelphia: Lippincott Williams and Wilkins; 2000.
20. Park IW, Lim OB, Park KN, Yi CH. Intra- and inter - rater reliability of measuring passive range of shoulder motion with smartphone and goniometer in patients with stroke. Phys Ther Korea 2014;21:1-12.

21. Yang H, Jeong C, Yoo Y, Bae S. Reliability study of hip range of motion measurement by smartphone inclinometer. J Korean Soc Integr Med 2016;4:89-96.

22. Kisner C, Colby LA, Borstad J. Therapeutic exercise: foundations and techniques. Philadelphia: F.A. Davis Company; 2017.

23. Cadet ER. Evaluation of glenohumeral instability. Orthop Clin North Am 2010;41:287-95.

24. Kolber MJ, Saltzman SB, Beekhuizen KS, Cheng MS. Reliability and minimal detectable change of inclinometric shoulder mobility measurements. Physiother Theory Pract 2009;25:572-81.

25. Provencher CM, Makani A, McNeil JW, Pomerantz ML, Golijanin P, Gross D. The role of the scapula in throwing disorders. Sports Med Arthrosc Rev 2014;22:80-7.

26. Choung SD, Weon JH, Jung DY. Effect of movement plane and shoulder flexion angle on scapular upward rotator during scapular protraction exercise. J Korean Soc Phys Med 2013;8:41-8.

27. Ha SM, Kwon OY, Cynn HS, Lee WH, Park KN, Kim SH, et al. Comparison of electromyographic activity of the lower trapezius and serratus anterior muscle in different arm-lifting scapular posterior tilt exercises. Phys Ther Sport 2012;13:227-32.

28. Ludewig PM, Hoff MS, Osowski EE, Meschke SA, Rundquist PJ. Relative balance of serratus anterior and upper trapezius muscle activity during push-up exercises. Am J Sports Med 2004;32: 484-93.

29. Park KM, Cynn HS, Yi CH, Kwon OY. Effect of isometric horizontal abduction on pectoralis major and serratus anterior EMG activity during three exercises in subjects with scapular winging. J Electromyogr Kinesiol 2013;23:462-8. 